\title{
Finite Element Modeling of the Cyclic Wetting Mechanism in the Active Part of Wheat Awns
}

\author{
Gerald A. Zickler • Davide Ruffoni · John W. C. Dunlop • \\ Rivka Elbaum • Richard Weinkamer · Peter Fratzl • \\ Thomas Antretter
}

Received: 20 April 2012/ Accepted: 15 June 2012/Published online: 12 July 2012

(C) The Author(s) 2012. This article is published with open access at Springerlink.com

\begin{abstract}
Many plant tissues and organs are capable of moving due to changes in the humidity of the environment, such as the opening of the seed capsule of the ice plant and the opening of the pine cone. These are fascinating examples for the materials engineer, as these tissues are non-living and move solely through the differential swelling of anisotropic tissues and in principle may serve as examples for the bio-inspired design of artificial actuators. In this paper, we model the microstructure of the wild wheat awn (Triticum turgidum ssp. dicoccoides) by finite elements, especially focusing on the specific microscopic features of the active part of the awn. Based on earlier experimental findings, cell walls are modeled as multilayered cylindrical tubes with alternating cellulose fiber orientation in successive layers. It is shown that swelling upon hydration of this system leads to the formation of gaps between the layers, which could act as valves, thus enabling the entry of water into the cell wall. This supports the hypothesis that this plywood-like arrangement of
\end{abstract}

\footnotetext{
G. A. Zickler $(\bowtie) \cdot$ T. Antretter

Institute for Mechanics, Montanuniversitaet Leoben, 8700 Leoben, Austria

e-mail: gerald.zickler@unileoben.ac.at

D. Ruffoni

Institute for Biomechanics, ETH Zurich,

8093 Zurich, Switzerland

J. W. C. Dunlop · R. Weinkamer · P. Fratzl

Department of Biomaterials, Max Planck Institute

of Colloids and Interfaces, 14424 Potsdam, Germany

R. Elbaum

Robert H. Smith Institute of Plant Science and Genetics

in Agriculture, Hebrew University of Jerusalem,

76100 Rehovot, Israel
}

cellulose fibrils enhances the effect of ambient humidity by accelerated water or vapor diffusion along the gaps. The finite element model shows that a certain distribution of axially and tangentially oriented fibers is necessary to generate sufficient tensile stresses within the cell wall to open nanometer-sized gaps between cell wall layers.

\section{Introduction}

Awns and appendages attached to seeds play an important role in dispersing the seed from the mother plant to the germination site [1-3]. Throughout millennia nature evolved various wings, hairs, and hooks for controlling the route of dispersal to a safe site. The seed has to be designed in such a way as to optimize its ability to find proper conditions for germination. Therefore, seeds bearing active awns are more abundant in structured soils, i.e. soils containing pores and aggregates of different sizes [3]. Seeds with hygroscopically active awns are able to propel themselves below the ground by coiling and uncoiling of their awns [4]. The mechanism of hygroscopic movement is a consequence of wetting and drying of tissue, which results in different anisotropic swelling in different regions of the tissue. Humidity-driven movement is not only shown in wheat awns, it is also reported for various other species [5-9]. This movement is interesting for the (bio)materials engineer as the movement occurs without an active metabolism and as such is controlled solely by the architectural arrangement of the different swellable tissues.

The present study focuses on the seed of the wild wheat plant (Triticum turgidum ssp. dicoccoides), which is attached to two large pronounced awns (Fig. 1a). Their cross-section consists macroscopically of three parts, i.e. active part, resistance part, and intermediate gap (Fig. 1b). 


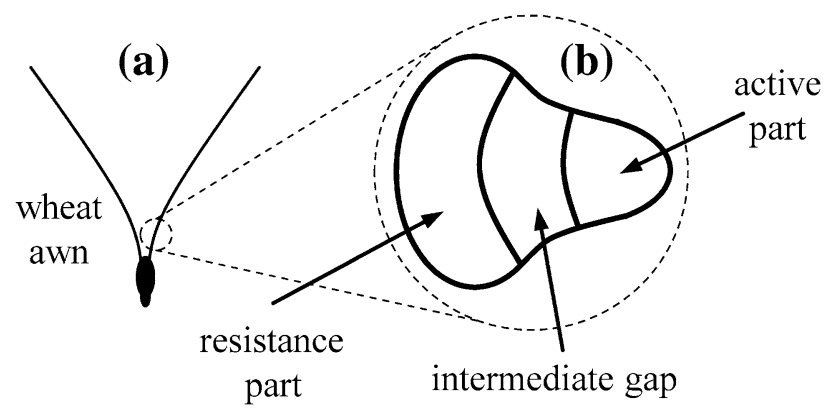

Fig. 1 a General overview of the dispersal unit of the wild wheat plant, consisting of the seed case and two pronounced awns. b Schematic cross-section of the lower part of the awn, consisting of the resistance part, the intermediate gap, and the active part

The active part (responsible for the awn bending with changing air humidity $[4,10])$ is built of cylindrical cells containing layers with parallel cellulose fibrils embedded in a soft hygroscopic matrix (Fig. 2). Neighboring layers are rotated with respect to each other, resulting in a nearly isotropic distribution of fibril directions when averaged over the entire thickness of the cell wall. Plywood architectures are very common as strategies for reinforcement of plants [11-13], bone [11, 14-16], collagen [17], and chitin [18, 19]. Although consisting only of dead tissue, wheat awns work like muscles, being able to transport seeds away from their host. The active part of the awn reacts to humidity changes, i.e. it elongates when wet and contracts when dry. In the case of the wheat awn, the matrix around cellulose fibrils swells with increasing air humidity, and the cellulose fibril direction controls the actual deformation, where the main direction of swelling is perpendicular to the direction of the cellulose fibrils.

In a previous study on the structure of the active part of the wheat awns, Elbaum et al. [10] speculated that the alternating orientation of cellulose in successive cell wall layers might enhance vapor (or water) diffusion if gaps opened between every second layer in the plywood structure. This somewhat counter intuitive mechanism, that

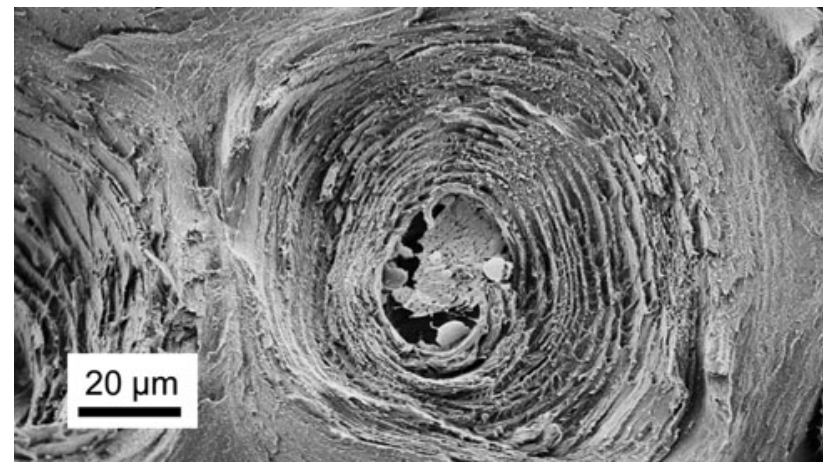

Fig. 2 Environmental scanning electron micrograph of a cell in the active part of the wheat awn showing the alternating layered structure swelling may lead to opening pores, was inspired by the idea that swelling of the layer in circumferential direction would be strongly reduced in the layers where the cellulose runs circumferentially, but not in layers where the cellulose runs axially. However, a quantitative estimate whether this effect is actually possible was not given in this experimental study.

The present work endeavors to study the proposed mechanism of humidity-driven movement and to find out whether, and under which conditions, an opening of gaps between cell wall layers is possible through swelling. The wheat awn is modeled using finite elements based on the structure reported in the earlier electron microscopic study [10]. The focus is on a mechanical description of the wetting mechanism. The interface between the various cell wall layers is monitored depending on their relative thickness and their cellulose orientation. Finally the composite material properties of such a cell are used to model the motion of the entire awn, which is validated by comparison with experimental results of awn bending as a function of humidity.

\section{Modeling}

\subsection{Macroscopic Modeling}

The aim of the macroscopic modeling is the determination of the expansion coefficient $\alpha$ due to moisture swelling. The expansion coefficient was obtained by means of finite element analysis by imposing the curvature of the finite element model to be equal to the curvature of the awn (Fig. 1a) measured experimentally under varying humidity conditions. The geometry of a representative cross-section of the awn for the finite element model came from scanning acoustical microscopy (SAM) images [4]. Based on the gray level, three distinct regions were manually identified: the active part, the intermediate gap, and the resistance part (Fig. 1b). The elastic moduli were taken from a previous study on the wheat awn, where Elbaum et al. determined $E_{\mathrm{a}}^{*}=10.0 \pm 2.8 \mathrm{kN} \mathrm{mm}^{-2}$ for the active part and $E_{\mathrm{r}}^{*}=20.5 \pm 2.6 \mathrm{kN} \mathrm{mm}^{-2}$ for the resistance part by nanoindentation [4]. The elastic modulus for the intermediate gap could not be measured directly, thus it was obtained by interpolation between the data of the resistance part and active part based on gray scale intensity of the SAM images. Local mean gray levels from SAM images were taken from all three regions, hence a linear interpolation provides $E=0.79 \mathrm{kN} \mathrm{mm}^{-2}$ for the intermediate gap region. The active part and the intermediate gap are composed of an alternating sequence of strongly anisotropic layers due to the direction of the fibrils. However, the 
ensemble properties are more or less isotropic for this very special arrangement. In the resistance part, on the other hand, all these fibrils are uniformly oriented in axial direction [4], thus for this part a special case of orthotropytransversally isotropic material properties $[20,21]$-were chosen.

A two-dimensional model of the cross-section was built of generalized plane strain elements (CPEG8) using the finite element software Abaqus [22]. The swelling due to a humidity change of $10 \%$ was mimicked by thermal expansion caused by a positive temperature step of $\Delta T=1 \mathrm{~K}$. The expansion was assumed isotropic in the active part, orthotropic in the resistance part and no expansion in the gap. Thus, the wheat awn deforms to a circular shape with constant curvature when the individual parts of the cross-section expand or contract.

\subsection{Microscopic Modeling}

Microscopic properties were studied by finite element modeling of the cylindrical cells of the active part. The active part of the wheat awn was built of an arrangement of longitudinally oriented cylindrical cells $[4,10]$. These cylindrical cells were made of cellulose fibrils arranged in layers with preferred axial and tangential directions [10]. Figure 3a shows a sketch of one single cylindrical cell built of several layers with alternating orientation of cellulose fibrils, wrapped around a cylindrical cavity. The cross-section of the cell is schematically drawn in Fig. 3b, showing the various layers of cellulose fibrils.

The single cylindrical cell of the active part was modeled as a plywood architecture with the finite element software Abaqus [22]. Based on scanning electron microscopy images [10], the inner radius $r_{\mathrm{i}}$ was set to $5 \mu \mathrm{m}$ and the outer radius $r_{\mathrm{o}}$ to $7 \mu \mathrm{m}$. The cylindrical cell was (a)

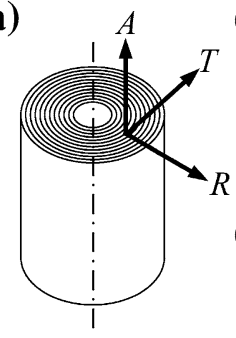

cylindrical cell (b) radial section

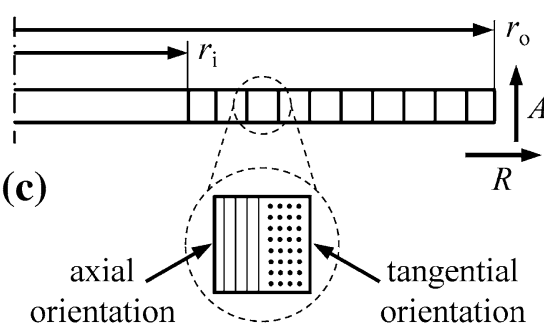

of cellulose fibrils
Fig. 3 a Schematic drawing of one single cylindrical cell of the active part containing several layers of oriented fibrils, with axial $(A)$, circumferential or tangential $(T)$ and radial $(R)$ directions. b Radial section of the cell showing the different rings. c Each ring is composed of two sublayers with axially and tangentially oriented cellulose fibrils, respectively assumed to be composed of ten concentric rings with radial thickness of $0.2 \mu \mathrm{m}$ each. Each individual ring was set to contain two sublayers of material with axial and tangential cellulose orientation (Fig. 3c), and transversely isotropic material properties were assigned to both sublayers.

Transverse isotropy $[20,21]$ is a special subclass of orthotropy, which is characterized by a plane of isotropy at every point in the material. Assuming the 1-2 plane to be the plane of isotropy at every point, transverse isotropy requires $E_{1}=E_{2}=E_{\mathrm{p}}, v_{31}=v_{32}=v_{\mathrm{tp}}, v_{13}=v_{23}=v_{\mathrm{pt}}$, and $G_{13}=G_{23}=G_{\mathrm{t}}$, where $p$ and $t$ stand for in-plane and transverse directions, respectively. This means that for the sublayers where the cellulose is oriented axially, the 1-2 plane corresponds to the $R-T$ plane (and 3 is axial, $A$ ), while for those where the cellulose is oriented circumferentially, the 1-2 plane is $A-R$ (and 3 is tangential, $T$ ). While $v_{\text {tp }}$ has the physical interpretation of the Poisson's ratio that characterizes the strain in the plane of isotropy resulting from stress normal to it, $v_{\mathrm{pt}}$ characterizes the transverse strain in the direction normal to the plane of isotropy resulting from stress in the plane of isotropy. In general, the quantities $v_{\mathrm{pt}}$ and $v_{\mathrm{tp}}$ are not equal and are related by $v_{\mathrm{tp}} / E_{\mathrm{t}}=v_{\mathrm{pt}} / E_{\mathrm{p}}$. For transversely isotropic materials Hooke's law in Voigt-Nye notation reduces to [22]

$$
\begin{aligned}
\left(\begin{array}{c}
\varepsilon_{11} \\
\varepsilon_{22} \\
\varepsilon_{33} \\
\gamma_{12} \\
\gamma_{13} \\
\gamma_{23}
\end{array}\right)= & \left(\begin{array}{cccccc}
1 / E_{\mathrm{p}} & -v_{\mathrm{p}} / E_{\mathrm{p}} & -v_{\mathrm{tp}} / E_{\mathrm{t}} & 0 & 0 & 0 \\
-v_{\mathrm{p}} / E_{\mathrm{p}} & 1 / E_{\mathrm{p}} & -v_{\mathrm{tp}} / E_{\mathrm{t}} & 0 & 0 & 0 \\
-v_{\mathrm{pt}} / E_{\mathrm{p}} & -v_{\mathrm{pt}} / E_{\mathrm{p}} & 1 / E_{\mathrm{t}} & 0 & 0 & 0 \\
0 & 0 & 0 & 1 / G_{\mathrm{p}} & 0 & 0 \\
0 & 0 & 0 & 0 & 1 / G_{\mathrm{t}} & 0 \\
0 & 0 & 0 & 0 & 0 & 1 / G_{\mathrm{t}}
\end{array}\right) \\
& \times\left(\begin{array}{c}
\sigma_{11} \\
\sigma_{22} \\
\sigma_{33} \\
\tau_{12} \\
\tau_{13} \\
\tau_{23}
\end{array}\right),
\end{aligned}
$$

where $G_{\mathrm{p}}=E_{\mathrm{p}} / 2\left(1+v_{\mathrm{p}}\right)$ and the total number of independent constants reduces to five. The five constants were set to the following values: The elastic modulus in transverse direction $E_{\mathrm{t}}$, was set to $20 \mathrm{kN} \mathrm{mm}^{-2}$ according to Ref. [4], the in-plane elastic modulus $E_{\mathrm{p}}$, was varied from 3.0 to $10 \mathrm{kN} \mathrm{mm}^{-2}$, the Poisson's ratios $v_{\mathrm{p}}$ and $v_{\mathrm{tp}}$ were assumed to be equal to 0.48 , which seems to be reasonable for a composite of biological polymers, and lastly the shear modulus $G_{\mathrm{t}}$ was approximated by the relation $G_{\mathrm{t}}=E_{\mathrm{t}} / 2\left(1+v_{\mathrm{p}}\right)$, resulting in a numerical value of $6.8 \mathrm{kN} \mathrm{mm}^{-2}$.

One single ring was built in total of 100 eight-node biquadratic continuum stress/displacement elements of type CAX8 [22]. Each of these elements must be given 
either axial or tangential material orientation where the latter forms the outer sublayer and the former the inner one. For each individual ring $i$, the fraction of axial oriented material $\phi_{\mathrm{i}}$ is given by $\phi_{\mathrm{i}}=\frac{A_{\mathrm{A}, \mathrm{i}}}{A_{\mathrm{A}, \mathrm{i}}+A_{\mathrm{T}, \mathrm{i}}}$, where $A_{\mathrm{A}, \mathrm{i}}$ and $A_{\mathrm{T}, \mathrm{i}}$ represent the sections of axially and tangentially oriented material, respectively (Fig. 4). $\phi_{i}$ can be varied between 0 and $100 \%$. The influence of vapor adsorption due to changes in humidity was replaced in the Abaqus formalism by a variation of temperature. The numerical value of the expansion coefficient of $\alpha=0.025 \mathrm{~K}^{-1}$ was a result of the macroscopic modeling (Sect. 2.1) to fit the macroscopic behavior of the wheat awn. The expansion coefficient $\alpha$ was assumed to behave transversely isotropic with $\alpha=0.025 \mathrm{~K}^{-1}$ in the plane of isotropy and no expansion perpendicular to it. It should be noted that both axially and tangentially oriented materials adsorb the same amount of water per unit volume and hence expand to the same extent, however anisotropically, i.e. in different directions. For the exact characterization of the ring arrangement two conditions had to be satisfied: First, the ensemble properties made up by the material data of the individual rings had to agree with the experiments, and second, the interfaces between the rings had to open upon swelling (so that the cell can operate as a valve). Such a geometry was found by following the procedure shown in Fig. 5. The ring arrangement was built from inside out, i.e. starting with the innermost ring with an initial guess for the volume fraction of axially oriented fibers $\phi_{1}$. This inner ring was subjected to a temperature change mimicking a humidity change which in turn entails a diameter growth of the entire ring. Now the second ring had to be designed. In the initial, undeformed state the inner radius of the second ring exactly matches the outer radius of the first ring, i.e. the rings just touch each other. However, the volume fraction of axially oriented fibers of the second ring $\phi_{2}$ had to be

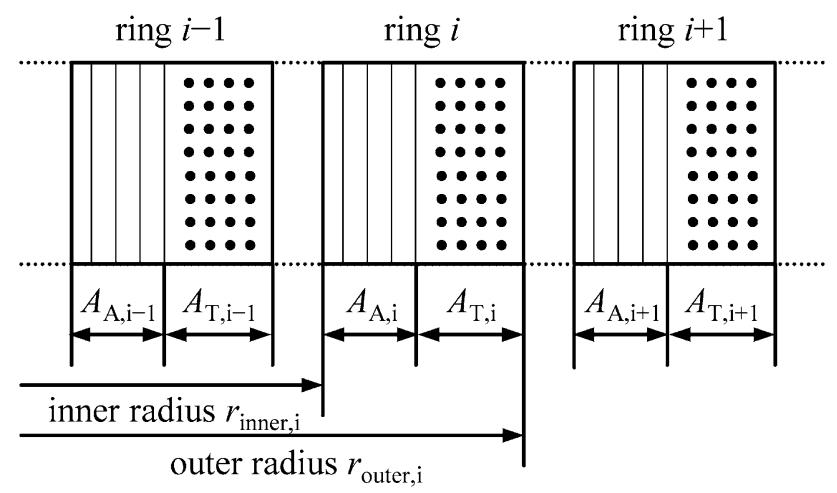

Fig. 4 Schematic composition of individual rings, showing the sections of axially $A_{\mathrm{A}, \mathrm{i}}$ and tangentially $A_{\mathrm{T}, \mathrm{i}}$ oriented material. The fraction of axially oriented material $\phi_{\mathrm{i}}$ for the ring $i$ is given by $\phi_{\mathrm{i}}=\frac{A_{\mathrm{A}, \mathrm{i}}}{A_{\mathrm{A}, \mathrm{i}}+A_{\mathrm{T}, \mathrm{i}}}$

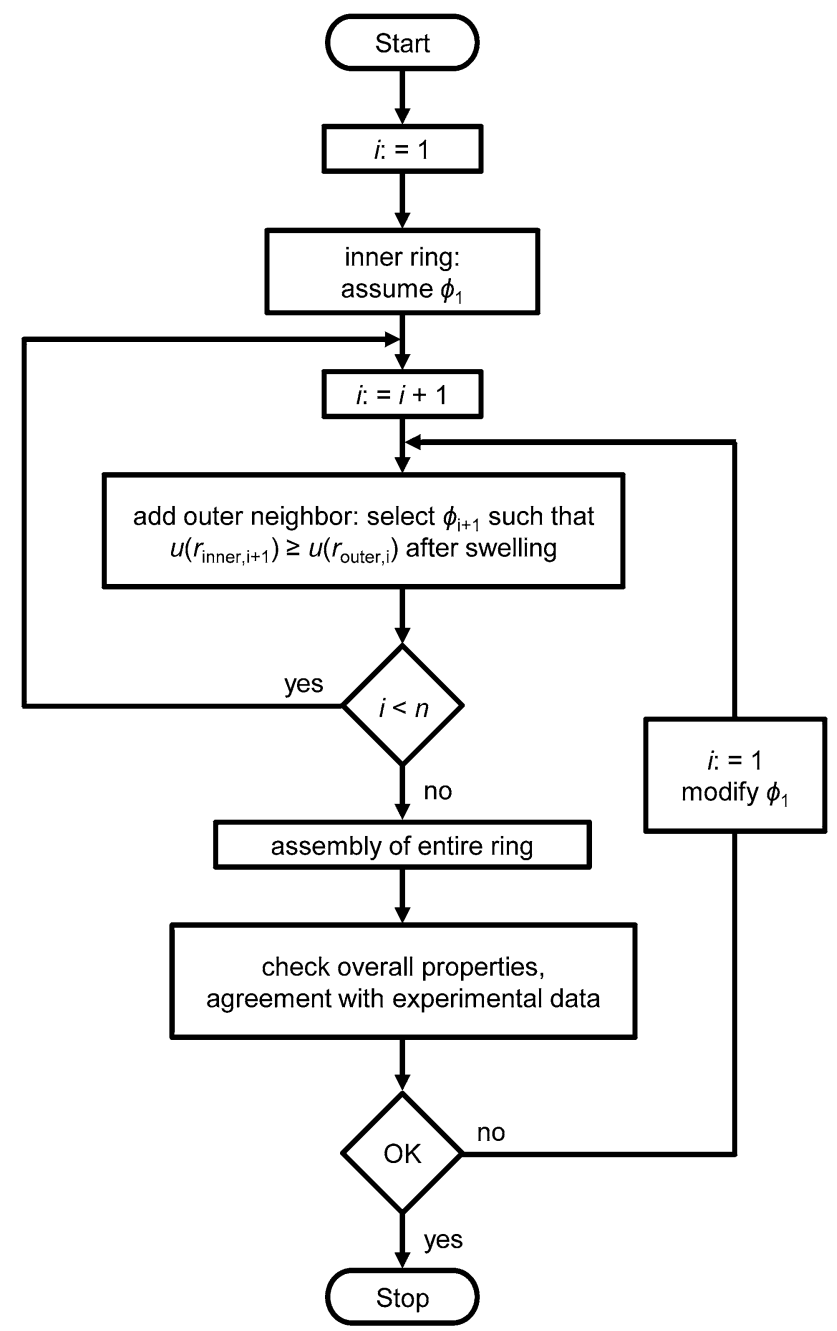

Fig. 5 Flowchart displaying the methodology for constructing a cell arrangement of a total number of rings $(n=10) . u$ denotes the radial displacement of an interface

chosen in such a way that after swelling the displacement $u$ at the inner radius of the second ring became slightly larger than the outer radius of the first ring leaving a gap at the interface. This procedure was repeated for the remaining rings $i=3, \ldots, 10$. In the end one had to double-check that the full assembly of rings still satisfies the conditions given above. While the presented procedure essentially guarantees the opening of the interfaces, the overall mechanical behavior had to be validated in a subsequent finite element calculation of the full ring assembly. Note that the full model represents a slice of a cell whose length dimension is significantly larger than its diameter. This corresponds to a generalized plane strain state which is accounted for by making sure that that model remains evenly bounded after deformation. The thickness change of the slice was recorded and related to an applied external force yielding a stiffness measure (essentially representing the elastic moduli of the composite). Basically, the initial choice made 
for $\phi_{1}$ decides upon the final cell design and thus also on the total volume fraction of axially oriented fibers $\Phi$. Consequently, repeating this cell design procedure for varying values of $\phi_{1}$ produces cells with stiffnesses covering a wide range. The final cell geometry is selected so that it best agrees with the measurements reported in [4]. For a parameter study, the calculations were performed with in-plane elastic moduli $E_{\mathrm{p}}$ of $3.0,5.0,8.0$, and $10 \mathrm{kN} \mathrm{mm}^{-2}$, respectively.

It should be noted that the finite element model of the full assembly provides not only the elastic moduli of the composite and the Poisson's ratios of the total cylindrical cell depending on the composition of axially and tangentially oriented material and the in-plane elastic modulus $E_{\mathrm{p}}$, but also the distribution of stresses inside the rings, including the radial stress components.

\section{Results}

The macroscopic modeling of the wheat awn provides a thermal expansion coefficient $\alpha$ of $0.025 \mathrm{~K}^{-1}$ for a positive temperature step of $\Delta T=1 \mathrm{~K}$, which mimics swelling due to a humidity change of $10 \%$. The expansion coefficient $\alpha$ is used for microscopic modeling of the wheat awn. For the sake of clarity the following nomenclature is used: The fraction of axially oriented material of an individual ring can be varied from 0 to $100 \%$. The total fraction of axially oriented material $\phi$ describes the composition of the entire cell and can also be varied from 0 to $100 \%$.

Starting from the fraction of axially oriented material of the inner ring, following the procedure described in Sect. 2.2, a series of individually composed cells is investigated. It is found that the total fraction of axially oriented material $\Phi$ of the entire cell strongly depends on the fraction of

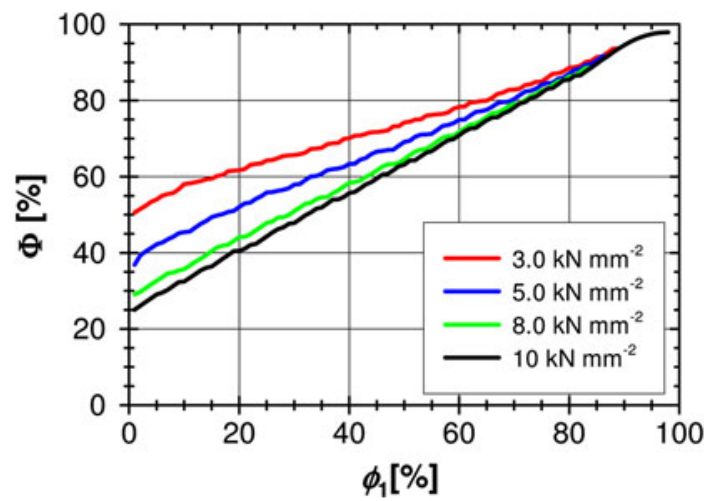

Fig. 6 The overall composition of the cell depends on the composition of the inner ring. Total fraction of axially oriented material $\Phi$ as a function of fraction of axially oriented material of the inner ring $\phi_{1}$ for various in-plane elastic moduli $E_{\mathrm{p}}$ (see legend) axially oriented material of the inner ring $\phi_{1}$ and the in-plane elastic modulus $E_{\mathrm{p}}$. It is shown in Fig. 6 that $\Phi$ increases with increasing fraction of axially oriented material of the inner ring $\phi_{1}$. The influence of $E_{\mathrm{p}}$ is strongest in combinations starting with small fractions of axially oriented material of the inner ring, i.e. for $E_{\mathrm{p}}=3.0 \mathrm{kN} \mathrm{mm}^{-2}$ the minimum value of $\Phi$ is about $50 \%$, whereas for $E_{\mathrm{p}}=10 \mathrm{kN} \mathrm{mm}^{-2}$ the minimum value of $\Phi$ reaches only about $25 \%$. At higher fractions of axially oriented material of the inner ring, $\Phi$ tends towards $100 \%$ for all four values of $E_{\mathrm{p}}$.

The fraction of axially oriented material governs the displacement of the individual rings. Thereafter the cells are built up of ten compatible rings (Sect. 2.2) and the mechanical behavior of the entire cell is calculated. Figure 7 shows a typical distribution of radial stresses $\sigma_{\mathrm{R}}$ along the cylindrical cell with $\Phi=80 \%$ after a positive temperature step of $1 \mathrm{~K}$. The numerical value of the in-plane elastic modulus $E_{\mathrm{p}}$ is set to $5.0 \mathrm{kN} \mathrm{mm}^{-2}$. It can be seen that the distribution has a saw-tooth shape with local maxima at the positions of interfaces between consecutive rings. One should keep in mind that in reality the stress distribution will not look like this, as gaps will open at the interfaces resulting in a redistribution of stresses. Nevertheless the stress distribution can be used as an indicator for choosing a very specific configuration, which fulfills the mechanism of a valve by opening gaps at the interfaces.

For a quantitative analysis of radial stresses, the absolute maximum value of $\sigma_{\mathrm{R} \text {,max }}$ within a distribution (Fig. 7) is plotted as a function of $\Phi$ for various in-plane elastic moduli $E_{\mathrm{p}}$ in Fig. 8a. It should be noted that Fig. 8 has been generated from a series of calculations, each giving a data point picked from a stress distribution a shown in Fig. 7. Therefore, one cannot expect these curves to be smooth. It is shown that $\sigma_{\mathrm{R}, \max }$ increases with increasing

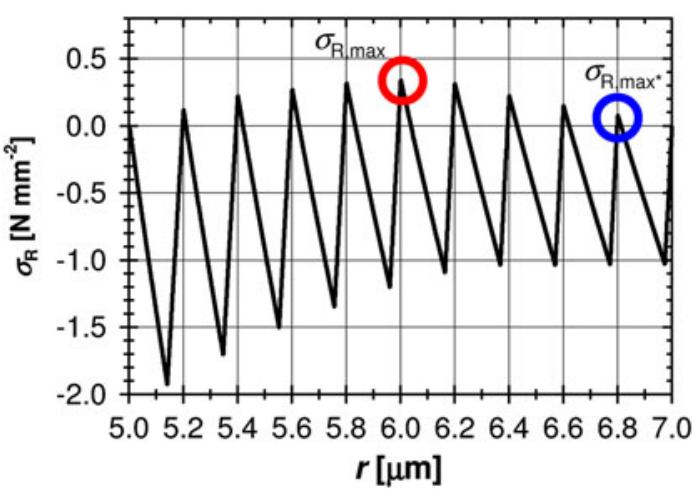

Fig. 7 Distribution of radial stresses $\sigma_{\mathrm{R}}$ along the radius $r$ of the cylindrical cell for a total composition of $\Phi=80 \%$ axial orientation after a positive temperature step of $1 \mathrm{~K}$. The numerical value of the in-plane elastic modulus $E_{\mathrm{p}}$ was set to $5.0 \mathrm{kN} \mathrm{mm}^{-2} . \sigma_{\mathrm{R}, \max }$ indicates the absolute maximum radial stress and $\sigma_{\mathrm{R}, \text { max}^{*}}$ represents the minimal maximum radial stress 


\section{$\Phi[\%]$}
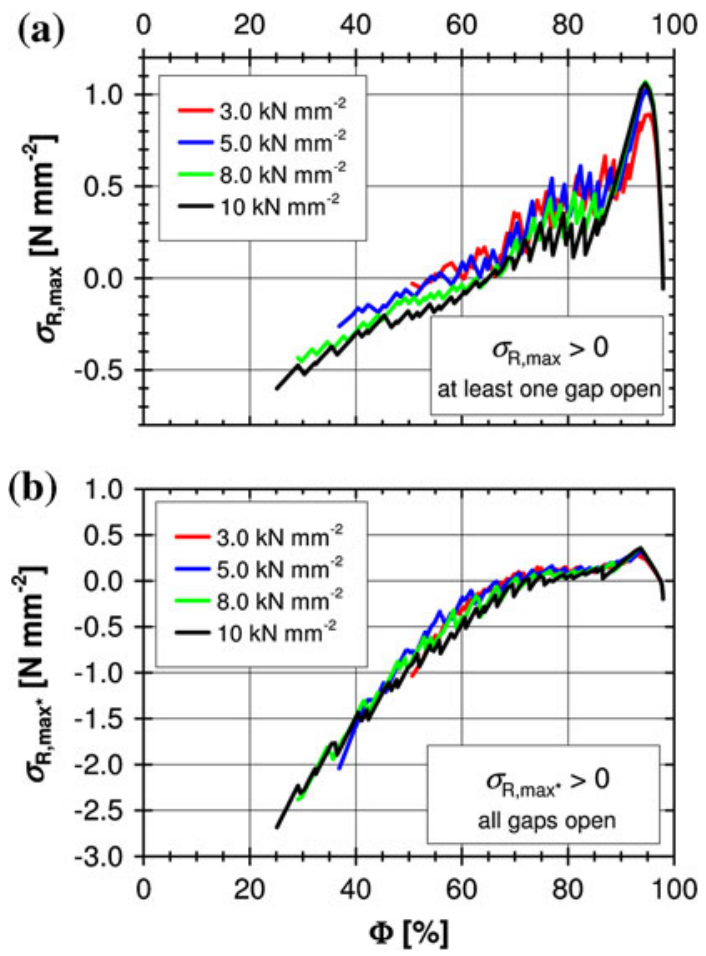

Fig. 8 a Absolute maximum radial stress $\sigma_{\mathrm{R}, \max }$ as a function of total fraction of axially oriented material $\Phi$ after a positive temperature step of $1 \mathrm{~K}$ for various in-plane elastic moduli $E_{\mathrm{p}}$ (see legend). For combinations with $\sigma_{\mathrm{R}, \max }>0$ at least one gap opens. b Minimal maximum radial stress $\sigma_{\mathrm{R}, \max *}$ as a function of total fraction of axially oriented material. For combinations with $\sigma_{\mathrm{R}, \text { max}^{*}}>0$ all gaps open

fraction of axially oriented material, however, not all combinations reveal positive values of $\sigma_{\mathrm{R}, \max }$. The crossover from negative to positive $\sigma_{\mathrm{R}, \max }$ depends on the in-plane elastic modulus and shifts to higher values of $\Phi$ with increasing $E_{\mathrm{p}}$.

However, one should keep in mind that for combinations with $\sigma_{\mathrm{R}, \max }>0$ (Fig. 8a) at least one interface senses tensile stresses. For determining combinations that sense tensile stresses at each interface Fig. $8 \mathrm{~b}$ shows the minimal value of the local maximum radial stress $\sigma_{\mathrm{R}, \max *}$ (Fig. 7) at the interfaces as a function of $\Phi$. It is seen that the fraction of crossover is further shifted towards higher values of $\Phi$. Most interestingly, all four curves almost overlap, meaning that there is hardly any influence of the in-plane elastic modulus.

In a subsequent finite element modeling step the elastic moduli $E_{\mathrm{A}}$ for the entire cell in axial direction and its Poisson's ratios $v_{\mathrm{TR}}$ are calculated for isothermal conditions as functions of $\Phi$ for various in-plane elastic moduli. Figure $9 \mathrm{a}$ shows $E_{\mathrm{A}}$ as a function of $\Phi$ for various $E_{\mathrm{p}}$, where it can be seen that $E_{\mathrm{A}}$ increases fairly linearly with
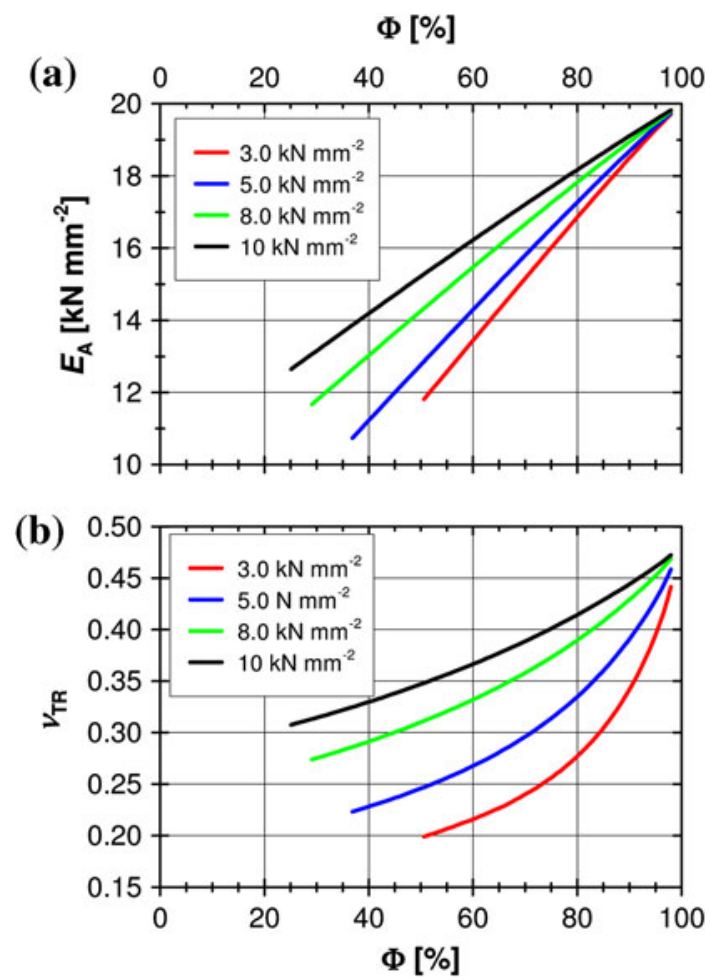

Fig. 9 a Elastic moduli $E_{\mathrm{A}}$ for the axial direction of the cell and $\mathbf{b}$ Poisson's ratios $v_{\mathrm{TR}}$ as a function of total fraction of axially oriented material $\Phi$ for various in-plane elastic moduli $E_{\mathrm{p}}$ (see legend)

increasing $\Phi$ up to $20 \mathrm{kN} \mathrm{mm}^{-2}$, which is the numerical value of the transverse elastic modulus $E_{\mathrm{t}}$. The lower values of $E_{\mathrm{A}}$ are located in the rather tight range between 11 and $13 \mathrm{kN} \mathrm{mm}^{-2}$, depending on $E_{\mathrm{p}}$. As shown in Fig. $9 \mathrm{~b}$, the Poisson's ratio $v_{\mathrm{TR}}$ of the whole cell depends on $\Phi$ and $E_{\mathrm{p}}$, i.e. the value of $v_{\mathrm{TR}}$ increase with $\Phi$ and is higher for higher $E_{\mathrm{p}}$.

It should also be mentioned that the numerical values of the shear modulus $G_{\mathrm{t}}$ does not have any influence, neither on the composition of the cell nor on the elastic modulus $E_{\mathrm{A}}$, and variations of $v_{\mathrm{p}}$ and $v_{\mathrm{tp}}$ show only minor influence on the calculated parameters.

\section{Discussion}

The microscopic finite element modeling of the active part provides valuable information on the possible mechanism of humidity-driven movement of the wheat awn. Although it is clear that differential swelling in different regions of a tissue leads to bending, one issue that is not often addressed is a question of kinetics and how water (vapor) can penetrate deep into the tissues. In a previous study on the wheat awn, Elbaum et al. [10] proposed that water transport into the active part of the awn is enhanced due to swelling itself, 
where tensile stresses at the interface between consecutive layers result in the formation of gaps, thus acting as valves, enabling a faster entry of water into the cell wall. The present study has investigated this model numerically and gives deeper insight into mechanisms of how the complex structure of the cell wall can lead to relatively fast motion or actuation. The selection of proper modeling parameters is done under two constraints: (a) All interfaces between two rings must open and (b) the total elastic modulus $E_{\mathrm{A}}$ should be in the range of experimental data given in Ref. [4], see Sect. 1. As shown in Fig. 8b, only cells with volume fractions greater than about $65 \%$ experience tensile rather than compressive stresses at each interface position, indicating that the gaps will open. This value is quite independent of $E_{\mathrm{p}}$, however, the elastic modulus $E_{\mathrm{A}}$ of the entire cell depends on $E_{\mathrm{p}}$ (Fig. 9a). Therefore, $E_{\mathrm{p}}=5.0 \mathrm{kN} \mathrm{mm}^{-2}$ seems to be reasonable, which results in an overall elastic modulus $E_{\mathrm{A}}$ of about $15 \mathrm{kN} \mathrm{mm}^{-2}$. This combination fulfills constraint (a), but is slightly larger than the experimental value $E_{\mathrm{a}}^{*}=10.0 \pm 2.8 \mathrm{kN} \mathrm{mm}^{-2}$ for the active part determined by nano-indentation [4]. However, it should be taken into account that nanoindentation provides an indentation modulus under the assumption of isotropic material behavior. Furthermore, when comparing experimental and calculated values, one should keep in mind possible effects of porosity and cleavage due to the penetration of a sharp-edged diamond pyramid in a rather soft fiber composite material. Moreover, one should consider that the elastic modulus depends on the water content which changes during movement. The mechanical response of the cell wall is also likely to depend on the environmental humidity [23, 24], although — to simplify the analysis in the current paper-a constant modulus was assumed.

Figure 10 shows the distribution of axially oriented material for all ten rings, which fulfills both constraints (a) and (b) given above. The fraction of axially oriented material $\phi_{\mathrm{i}}$ increases from $45 \%$ in the inner ring to $79 \%$ in the outer ring, resulting in a total volume fraction of $66 \%$. The increase of the axial fraction approximately follows a square-root function. It should be emphasized that this square-root dependence is a result of the procedure described in Sect. 2.2. It is a topic of ongoing research if this finding can be verified experimentally. Of course, one could ask for other possible distributions, which are also able to fulfill the constraints. For the sake of comparison, the distributions of radial stresses $\sigma_{\mathrm{R}}$ are calculated for two additional cases with identical volume fraction, i.e. (1) constant distribution and (2) linear distribution with a positive gradient. The distribution of radial stress $\sigma_{\mathrm{R}}$ in the composed cell gives an indication whether the cell can actually act as a valve. Tensile stresses are required for the

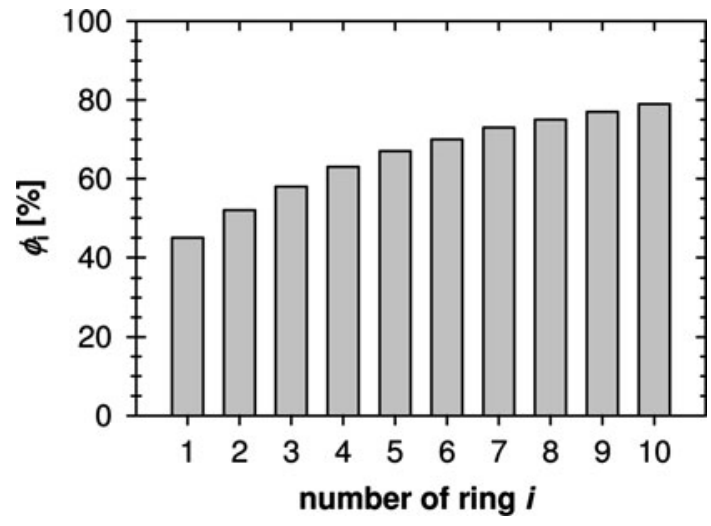

Fig. 10 Distribution of the fraction of axially oriented material $\phi_{\mathrm{i}}$ for each individual ring $i$. The numerical value of in-plane elastic modulus $E_{\mathrm{p}}$ is set to $5.0 \mathrm{kN} \mathrm{mm}^{-2}$. For the presented combination, the total fraction of axial material $\Phi$ is about $66 \%$, the axial elastic modulus $E_{\mathrm{A}}$ is about $15 \mathrm{kN} \mathrm{mm}^{-2}$ and all gaps between two individual rings

gaps to open, whereas compressive stresses show that the gaps are still closed. The results of the composed distribution (Fig. 10) and the two additional distributions with identical volume fractions are displayed in Fig. 11. It is demonstrated that only the square-root-shaped distribution is able to generate equivalent tensile stresses at each interface position. By contrast the constant distribution results in compression stresses at the interfaces. For the gradient distribution the outer interfaces sense rather high tensile stresses, whereas the inner ones are under compression. This finding shows that the proposed mechanism of gap opening can only be realized by a distribution with the shape of a square-root function (Fig. 10).

The model proposed in Ref. [10] suggests that the swelling of alternating layers of axially and tangentially oriented tissues leads to the opening of nanometer-sized gaps at the interface positions between the consecutive

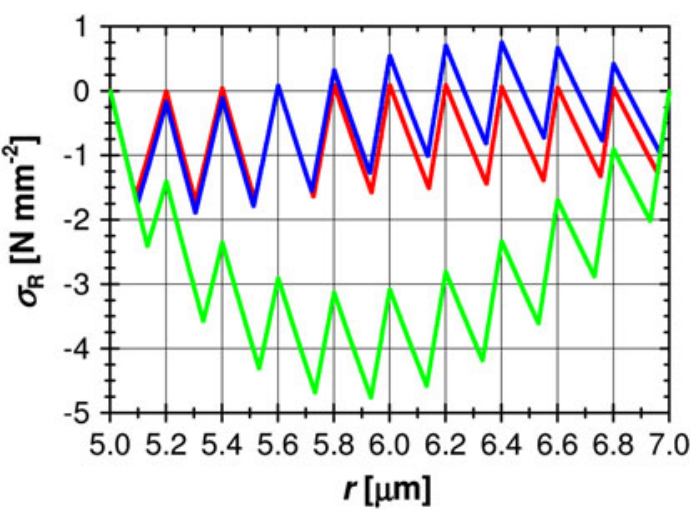

Fig. 11 Radial distribution of stresses $\sigma_{\mathrm{R}}$ along the radius $r$ of the cylindrical cell for the composition shown in Fig. 10 (red line). For comparison, two additional distributions with identical fraction of axially oriented material are shown: linear distribution with positive gradient (blue line) and constant distribution (green line) 


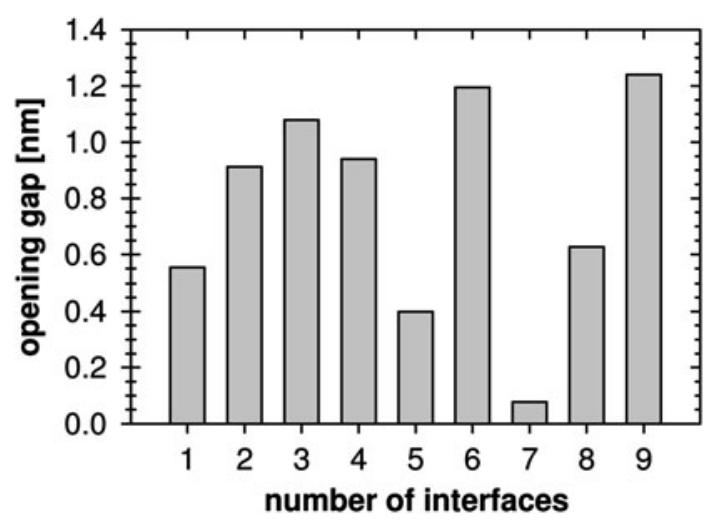

Fig. 12 Opening gaps at the interfaces between individual rings for ten individual rings with the composition presented in Fig. 10 after a positive temperature step of $1 \mathrm{~K}$. The numbering of the interfaces starts from the core of the cell

layers and is supported by the present study. Figure 12 shows the opening gaps at the interfaces between individual rings for a combination of ten rings with compositions presented in Fig. 10. It is shown that each interface between two rings opens to a nanometer-sized gap, which would work like a valve for water transport into the active part of the wheat awn. The effect of irregular opening can be accounted for by the discrete character of the applied finite element model, which currently does not provide the possibility to continuously vary the fraction of axially and tangentially oriented material. Nevertheless, the presented numerical model is a valuable tool for understanding the mechanism of water transport in plants showing humiditydriven movements.

\section{Summary and Conclusions}

In the present study the microstructure of the wild wheat awn (Triticum turgidum ssp. dicoccoides) is modeled by finite elements, especially focusing on the specific microscopic features of the active part. Depending on its composition the nano-scaled plywood architecture of the active part senses tensile stresses at the interfaces of individual rings of arranged fibrils. These stresses result in the formation of gaps, thus acting as valves, to allow faster entry of water into the cell wall. This is a somewhat counterintuitive mechanism, where swelling of the plywood-like fibril arrangement of tissue leads to the formation of pores. It may therefore play a role in enhancing the rate at which changes in ambient humidity lead to swelling of the tissue, thus enabling the actuation of the awn under moderate humidity changes. The finite element model shows that only selected distributions of axially and tangentially oriented fibers are able to generate tensile stresses within the cell wall, which lead to the opening of nanometer-sized gaps. Constant distributions and linearly increasing distributions fail to generate the effect of constant opening over the entire cross-section of the cell. The overall elastic modulus is in reasonable agreement with experimental data from nano-indentation probing on wheat awns of the same species. The homogeneous properties of the tissue are also consistent with simple calculations of awn curvatures at the macroscopic scale with changes in humidity.

The present study has shown that a very special layering of the cells constituting the wheat awns is required to serve two purposes: (a) Provide sufficient stiffness to guarantee to work as an efficient actuator and (b) act as a humiditydriven valve to enable a sufficient amount of water intake. The presented concept involving experimental techniques in combination with numerical methods has proven successful for the quantification of experimentally inaccessible values in natural tissue.

The next logical step is to take the findings for the material behavior on the cell scale to the next hierarchical level of the entire wheat awn by again employing the finite element method in combination with the existing experimental evidence.

Open Access This article is distributed under the terms of the Creative Commons Attribution License which permits any use, distribution, and reproduction in any medium, provided the original author(s) and the source are credited.

\section{References}

1. Harper JL, Lovell PH, Moore KG (1970) Annu Rev Ecol Syst $1: 327-356$

2. Peart MH (1981) J Ecol 69:425-436

3. van der Pijl P (1982) Principles of dispersal in higher plants. Springer, Berlin

4. Elbaum R, Zaltzman L, Burgert I, Fratzl P (2007) Science 316:884-886

5. Witztum A, Schulgasser K (1995) J Theor Biol 176:531-542

6. Dawson C, Vincent JFV, Rocca AM (1997) Nature 390:668

7. Reyssat E, Mahadevan L (2009) J R Soc Interface 6:951-957

8. Abraham Y, Tamburu C, Klein E, Dunlop JWC, Fratzl P, Raviv U, Elbaum R (2012) J R Soc Interface 9:640-647

9. Harrington MJ, Razghandi K, Ditsch F, Guiducci L, Rueggeberg M, Dunlop JWC, Fratzl P, Neinhuis C, Burgert I (2011) Nat Commun 2:337

10. Elbaum R, Gorb S, Fratzl P (2008) J Struct Biol 164:101-107

11. Fratzl P, Weinkamer R (2007) Prog Mater Sci 52:1263-1334

12. Fratzl P, Elbaum R, Burgert I (2008) Faraday Discuss 139:275-282

13. Fratzl P, Barth FG (2009) Nature 462:442-448

14. Weiner S, Arad T, Sabanay I, Traub W (1997) Bone 20:509-514

15. Weiner S, Traub W, Wagner HD (1999) J Struct Biol 126:241-255

16. Wagermaier W, Gupta HS, Gourrier A, Burghammer M, Roschger P, Fratzl P (2006) Biointerphases 1:1-5

17. Giraud-Guille MM (1998) Curr Opin Solid State Mater Sci $3: 221-227$ 
18. Raabe D, Sachs C, Romano P (2005) Acta Mater 53:4281-4292

19. Al-Sawalmih A, Li C, Siegel S, Fabritius H, Yi S, Raabe D, Fratzl P, Paris O (2008) Adv Funct Mater 18:3307-3314

20. Nye JF (1985) Physical properties of crystals. Oxford University Press, Oxford

21. Hull D, Clyne TW (1996) An introduction to composite materials. Cambridge University Press, Cambridge
22. Abaqus Analysis User's Manual (2012) Simulia, Dassault Systèmes, Vélizy-Villacoublay, France, http://www.simulia.com

23. Bodig J, Jayne BA (1993) Mechanics of wood and wood composites. Krieger Publishing, Malabar

24. Niemz P (1993) Physik des Holzes und der Holzwerkstoffe. DRW-Verlag, Stuttgart 